Full title: The Potential of Immune Biomarkers to Advance Personalized Medicine Approaches for Schizophrenia

Running title: Immune Biomarkers for Schizophrenia

David Cox MSc ${ }^{1}$, Man K. Chan PhD ${ }^{1}$, Sabine Bahn Prof ${ }^{1 *}$.

*Corresponding author. E-mail:sb209@cam.ac.uk

${ }^{1}$ Department of Chemical Engineering and Biotechnology, University of Cambridge, Cambridge, United Kingdom.

\title{
Acknowledgements
}

The authors gratefully acknowledge support by the Stanley Medical Research Institute. 


\section{The Potential of Immune Biomarkers to Advance Personalized Medicine Approaches for Schizophrenia}

\section{Abstract}

Molecular profiling studies have helped increase the understanding of the immune processes thought to be involved in the etiology and pathophysiology of psychiatric disorders such as schizophrenia. Current therapeutic interventions with first and second generation antipsychotics are suboptimal. Poor response rates and debilitating side effects often lead to poor treatment compliance. This highlights the pressing need to identify more effective treatments as well as objective biomarker based tests which can help predict treatment response and identify diagnostic subpopulations. Such tests could enable early detection of patients who will benefit from particular therapeutic interventions. In this review, we discuss studies relating to dysfunctions of the immune system in schizophrenia patients and the effects of antipsychotic medication on the molecular components of these systems. Immune system dysfunction may in part be related to genetic risk factors for schizophrenia, but there is substantial evidence that a wide range of environmental factors ranging from exposure to infectious agents such as influenza and Toxoplasma gondii to HPA axis dysfunction play an important role in the etiopathogenesis of schizophrenia. Ongoing research efforts, testing therapeutic efficacy of anti-inflammatory agents used as add-on medications are also discussed. From a therapeutic perspective these represent the initial steps towards novel treatment approaches and more effective patient care in the field of mental health.

Keywords: Schizophrenia; Biomarkers; Antipsychotic Medication; Personalized medicine 


\section{1: Introduction}

Schizophrenia is a major psychiatric disorder which affects up to $1 \%$ of the global population and has been listed among the world's top ten causes of disease-related disability (Tandon et al., 2008). It has an annual incidence rate of around 15 in every 100,000 people and the lifetime risk of developing the disease is approximately $0.7 \%$.

The development of effective treatments for schizophrenia patients has been hampered by a lack of significant innovations over the past 60 years since first-generation (typical) antipsychotics were introduced in the 1950s. First and second-generation (atypical) antipsychotics can be associated with poor response rates and prominent side effects.

As a result there is a great need to improve our understanding of the complex and diverse underlying pathophysiological mechanisms associated with the schizophrenia disease process at the molecular level. This improvement will enable treatment intervention at the earliest possible stage and increase our understanding of the mechanisms of action of antipsychotics.

Subgroups of schizophrenia patients may exist as some respond well to certain therapeutic compounds while others continue to experience severe and chronic symptoms (Tomasik et al., 2014). This distinction highlights a strong clinical need for robust biomarkers of treatment response which can enable the detection of patient subgroups and inform individual treatment strategies at an early stage.

This review will discuss the extent to which previous studies have increased our understanding of how "biomarkers" for schizophrenia are modulated by antipsychotic medication. In particular we will consider biomarkers involved in immune function which have been implicated in the disease process of schizophrenia. The review will also consider the potential use of combination therapies which can be used in early intervention, i.e. combining existing antipsychotics with adjunctive treatments such as anti-inflammatory agents. Initial studies have already provided promising results of increased efficacy in some patients. In the future it may be possible to apply these treatments to patient subgroups who display specific molecular traits.

\section{2: Immune System and Inflammatory Biomarkers}

\subsection{The immune system as a target for pharmacological interventions}

The immune system has been suggested as a novel therapeutic target for schizophrenia, due to evidence from neurobiological, genetic and environmental studies suggesting that alterations in immune function may be associated with the disease pathogenesis in at least a subgroup of patients (Potvin et al., 2008 and Chan et al., 2011). Schizophrenia patients and their relatives are often found to have a higher or lower than expected prevalence of auto-immune disorders such as autoimmune encephalitis, rheumatoid arthritis and celiac disease (Benros et al., 2011).

Genetics has provided considerable evidence that immune system dysfunction plays a key role in the pathophysiology of schizophrenia (Smyth and Lawrie, 2013). A 2013 study identified 144 genes relating to inflammation and immune response in the hippocampus of schizophrenia patients, which were differentially expressed compared to controls (Hwang et al., 2013). This study follows on from previous work identifying various gene loci on chromosome 6 as having the strongest association 
with the disease (Schizophrenia Psychiatric GWAS Consortium, 2011), a notable finding as this region includes a number of genes involved in immune function. Studies have also pointed to the potential harmful role of activated inflammatory processes in schizophrenia on the central nervous system (CNS) (Drexhage et al., 2011). These processes can result from a variety of factors including disease stage, infectious disease (as discussed further in section 3 ), the side effects of antipsychotic medication and other environmental risk factors. Inflammation represents a protective mechanism and this cellular response is controlled in both the periphery and CNS by immunomodulatory mechanisms through the up- or down-regulation of pro or anti-inflammatory cytokines/mediators and their receptors (Smyth and Lawrie, 2013).

Cytokines are soluble proteins secreted by a variety of different cells. Many cytokines are capable of passing the blood-brain barrier (BBB) and inducing changes in behaviour and cognition by altering brain function, for example through the hypothalamic-pituitary-adrenal (HPA) axis (Watanabe et al., 2010). This suggests that the CNS and the immune system are interconnected rather than being isolated as previously thought (Simard and Rivest, 2005). This interaction plays a key role in regulating dopaminergic and GABAergic neuronal differentiation as well as neuronal migration and synaptic maturation (Namba et al., 2006). Studies have suggested that processes leading to immune alterations in the CNS may originate in the periphery (Kirch et al., 1992). Altered levels of cytokines and other inflammatory markers have been found in schizophrenia patients in the blood and cerebrospinal fluid (Potvin et al., 2008, and Miller et al., 2011). These molecules can then cross the BBB and induce a variety of harmful consequences such as neuronal inflammation, damage and degeneration which have been implicated in the disease (Raison and Miller, 2011).

\subsection{Inflammatory markers and antipsychotic medication}

Cytokines are involved in the pathogenesis of schizophrenia and also the response to antipsychotic medication. In one meta analysis of 33 studies which examined peripheral immune alterations in schizophrenia (Miller et al., 2011), numerous biomarkers were reported as being elevated in first episode and relapsed patients, including plasma concentrations of cytokines Interleukin-1 beta (IL1ß), Interleukin-6 (IL-6) and Transforming growth factor beta (TGF- $\beta$ ). In particular, IL-6 levels were increased with an effect size of 1.4 in first episode patients. All three of these molecules were found to be normalized with treatment, suggesting they are state biomarkers. However Interleukin-12 (IL12), interferon gamma (IFN- $\gamma$ ), Tumor necrosis factor alpha (TNF- $\alpha$ ) and soluble interleukin-2 receptor (sIL-2R) were all increased in schizophrenia patients but were not altered by treatment, identifying them as possible trait biomarkers.

A number of additional candidate biomarkers have been reported to change in response to antipsychotic treatment, including S100B, prolactin, interleukin-2 (IL-2), insulin, leptin, interleukin-1 receptor antagonist (IL1-RA), interleukin-8 (IL-8), interleukin-2 receptor antagonist (IL-2RA) (Chan et al., 2011) and IL-6 which has been repeatedly reported to be increased in schizophrenia patients. These findings are in line with a recent 2008 meta analysis (Potkin et al., 2008) analyzing 62 studies of peripheral immune alterations in patients compared to controls. As this cytokine is predominantly produced by cells of the innate immune system, increased levels of IL- 6 point towards an abnormal innate immune response in these patients. The study also showed that IL-6 levels decreased after treatment with antipsychotics. However, it remains unclear whether these changes are primarily a result of medication or a change in the state of the disease. IL-6 levels have been shown to differ, 
depending on whether patients are in the acute state of illness or in remission, as evidenced by correlation with psychopathology scores (Pae et al., 2006). IL-6 upregulation is also only seen in some studies but not others (Maes et al., 2000).

Antipsychotics have been found to have differential effects on a variety of other pro-inflammatory cytokines. sIL-2R is a marker of immune activation, inhibiting IL-2 activity by preventing its binding to membrane-anchored receptors. Its levels are found to be increased above normal levels in schizophrenia patients (Potkin et al., 2008). However while studies have shown that sIL-2R and IL-12 increase further with antipsychotic treatment (Potkin et al., 2008 and Miller et al., 2011), the levels of other pro-inflammatory proteins IL-1 $\beta$ and IFN- $\gamma$ were found to decrease (Miller et al., 2011). This highlights the complexity of the relationship between antipsychotics and inflammation. The fact that the levels of some molecules indicate an excessive inflammatory state in the periphery may be due to the propensity of atypical antipsychotics to increase certain inflammatory processes, leading to side effects such as metabolic syndrome (Buchholz et al., 2008). Risperidone was found to have a particularly significant effect on increasing levels of IL-12 (Tourjman et al., 2013).

The peripheral levels of C Reactive Protein (CRP), a protein mainly generated in the liver, are also a good indicator of chronic inflammation with increased levels associated with infection and inflammatory conditions (Lowe, 2005). There have been limited studies examining this molecule in schizophrenia but recent data have shown that it is increased in around $30 \%$ of patients (Dickerson et al., 2013 and Miller et al., 2014). A 2010 study of 111 patients examined the effects of haloperidol, olanzapine and risperidone on CRP levels after three months and twelve months of treatment, respectively (Diaz et al., 2010). Haloperidol led to an increase in the levels of CRP after three months but there were no significant differences between baseline and twelve month levels for any of the treatments. This suggests the development of tolerance to the effects of antipsychotics on CRP levels but the results may be skewed by the fact that a number of patients had dropped out by the one year time point.

The levels of anti-inflammatory molecules, such as the cytokines IL1-RA and interleukin-10 (IL-10), have been found to increase in response to treatment (Potkin et al., 2008). Genetic studies have indicated that IL1-RA and IL-10 may play a causal role in the development of the disease through inhibiting normal cytokine action, resulting in altered brain and behavioural function (Dantzer, 2004). A recent study which looked at multiplex immunoassay analyses of serum cytokines in 180 first-onset schizophrenia patients before and after treatment with risperidone and olanzapine, found that the levels of both IL-10 and IL1-RA were significantly lower after six weeks of medication (De Witte et al., 2014). The decrease in the concentration of these anti-inflammatory cytokines is in line with the hypothesis that antipsychotics induce an inflammatory response which can lead to certain metabolic side-effects. Interestingly the decrease in IL-10 levels was also significantly correlated with an improvement in negative symptoms including cognitive functioning. This suggests that IL-10 may play an important role in the therapeutic mechanisms of these two atypical antipsychotic drugs. 


\subsection{Potential confounding effects of psychological stress and other factors}

The clinical implications of the findings relating to pro-inflammatory molecules such as IL- 6 suggest that a more individualized treatment approach may be appropriate for patients who display evidence of immune activation. For example, add-on anti-inflammatory agents could be used in conjunction with existing antipsychotics to target these molecules and their pathways (Keller et al., 2013). This approach is discussed in further detail in Section 4.

So far, a number of meta-analyses have been conducted to examine changes in inflammatory markers such as IL-6. However, many of these studies did not take into account methodological differences in storage, sample handling and methods of assessment, all of which can influence circulating cytokine levels. Consequently, further studies investigating cytokine responses in disease should account for the effects of various confounding factors. A few studies have examined some of the variables which may have a confounding effect on peripheral cytokine levels (Tappia et al., 1995). For instance, a 2013 meta analysis on the effects of antipsychotics on cytokines across 23 studies (Tourjman et al., 2013), found that age, gender and duration of illness had no effect on the results. However, other potential confounding factors such BMI, smoking and ethnicity were not considered.

A final limitation is the possibility that more complex mechanisms such as a reduction in psychological stress after treatment may result in the apparent reductions seen in the levels of some cytokines. Evidence for the influence of stress on cytokine levels has been provided by several studies. For instance, increased psychological stress in healthy individuals was found to induce inflammatory response resulting in increased production of pro-inflammatory cytokines such as TNF$\alpha$, IL-6, IL-1RA, IFN- $\gamma$ and IL-10 (Maes et al., 1998), and similar results have been obtained for other psychiatric disorders such as depression (Potkin et al., 2008). It has been hypothesized that this happens via pathways involving defective glucocorticoid-mediated feedback inhibition and exaggerated sympathetic nervous system-mediated activation of immune responses (Potkin et al., 2008).

\section{Infectious Agents}

\subsection{Evidence linking infectious agents to schizophrenia}

Exposure to pathogenic microbes, either during the prenatal period or childhood, has been suggested as being a contributing factor to the etiology of schizophrenia by causing disruptions in brain development (Murray and Lewis, 1987). Studies investigating the role of specific infectious agents in schizophrenia have suggested that the risk is mainly posed by viruses and parasites with a tendency to invade the central nervous system (CNS).

Over the past decade studies applying bioassays to measure anti-microbial antibodies in archived maternal serum samples have yielded some notable results. They suggest that infections posing a particular risk factor for schizophrenia include influenza A virus (Brown et al., 2004), polio virus (Suvisaari et al., 1999), rubella virus (Brown et al., 2000) and genital reproductive infections (Babulas et al., 2006), although the evidence surrounding herpes simplex virus type 2 is contradictory (Buka et al., 2008 and Brown et al., 2006). Influenza A virus has been suggested to increase the risk of 
developing schizophrenia by sevenfold when mothers are exposed to the virus during the first trimester of pregnancy (Brown et al., 2004).

Considerable research has been undertaken on the parasite Toxoplasma (T.) gondii and its potential contribution to the etiopathogenesis of schizophrenia (Mortensen et al., 2007 and Brown et al., 2005). Thirty eight studies have shown positive correlations between T. gondii antibody titers and schizophrenia, as of 2013 (Webster et al., 2013). Associations have been found between maternal and neonatal exposure to T.gondii and an increased risk to developing schizophrenia (Mortensen et al., 2007). T.gondii is of particular interest as links have also been observed between exposure in adulthood and schizophrenia in studies of the US military (Niebuhr et al., 2008) and ultra-high risk (UHR) individuals (Amminger et al., 2007).

A potential link has also been proposed between bacterial and viral meningitis in childhood and the onset of schizophrenia some years later (Leask et al., 2002).

However while associations have been made between schizophrenia and infections years to decades before diagnosis, investigations into how infectious agents contribute to the pathomechanisms of the disorder, after a lengthy latency period, are still ongoing.

\subsection{Evidence for biomarker changes associated with exposure to infectious agents}

One theory suggests infectious agents increase the risk of schizophrenia by altering the levels of cytokines and other immune mediators. These molecules play a critical role in how the immune system deals with infection, as well as being involved in the development and functioning of the CNS in early life. Some are pro-inflammatory, aiding the body deal with foreign pathogens and some are anti-inflammatory mediating the immune response and terminating it when no longer necessary (Parham, 2005 and Richard and Brahm, 2012).

An association has been found between increased levels of pro-inflammatory cytokines in maternal serum, as result of exposure to infection, and psychotic illness in the offspring (Brown et al., 2004). For instance maternally generated cytokines have been found to cross the placenta (Li et al., 1995) and partially penetrate the BBB of the developing fetus and bind to glial cells and neural receptors in the brain (Banks et al., 1994). Additionally, elevated levels of these molecules have also been shown to increase susceptibility to brain damage (Dammann and Leviton, 1997) through various mechanisms. One hypothesis is that this occurs through the disrupted maturation of oligodendrocytes, resulting in white matter abnormalities which have been seen in post-mortem brains and in imaging studies of schizophrenia patients (Davis et al., 2003). Cytokine activity can also induce other biological processes including increases in oxidative stress and activation of the hypothalamic-pituitary-adrenal (HPA) axis (Turnbull and Rivier, 1995).

Clinical studies have reported increases in the levels of several cytokines in the response to infection, notably IL-8 and TNF- $\alpha$ (Brown and Derkits, 2010). These molecules may be involved in cytotoxicity as well as mediating dopaminergic and glutamatergic pathways which are implicated in the pathophysiology of schizophrenia (Girgis et al., 2014).

Findings from animal models also suggest that IL-6 may play a key role in the development of schizophrenia-related phenotypes which occur as a result of maternal immune activation (MIA) (Smith et al., 2007). MIA was induced in pregnant rats by injecting a bacterial cell wall endotoxin 
called lipopolysaccharide (LPS) (Romero et al., 2007) and polyinosinic:polycytidylic acid (poly I:C). The latter imitates a viral infection by activating inflammatory signalling pathways through Toll-like receptor 3 via a nuclear factor KB-dependent mechanism (Smith et al., 2007) and stimulating cytokines such as IL-1, IL-6 and TNF- $\alpha$ (Gilmore et al., 2005). In both experiments IL- 6 levels were increased, which has been linked to cognitive deficits such as spatial memory and learning abnormalities in rodent models.

The potential pathophysiological consequences of infection in later life can be investigated through biomarkers related to infectious agents, some of which were reported to be correlated with functional deficits in schizophrenia. The levels of T.gondii immunoglobin $G$ (IgG) antibodies were found to be positively correlated with psychotic symptoms in ultra-high risk individuals (Amminger et al., 2007). A recent study which looked at molecular changes in the cerebrospinal fluid (CSF) in schizophrenia patients saw that T.gondii antibody levels were correlated with a disease associated reduction in TGF- $\alpha$, IL-6R and ACE (Hayes et al., 2014). Furthermore, antibodies to herpes simplex virus type 1 (HSV-1) have been obtained (Yolken et al., 2011). HSV-1 has been linked to impaired cognitive functioning (Prasad et al., 2012). A further study found that elevated serum levels of Creactive protein (CRP) along with exposure to HSV-1, is linked to the severity of cognitive impairment in schizophrenia (Dickerson et al., 2012).

\subsection{Anti-inflammatory/anti-microbial efficacy of antipsychotic medication}

The impaired cytokine signalling resulting from prenatal or childhood infection is believed to lead to abnormal brain development which ultimately may precipitate the onset of psychiatric symptoms post-adolescence. For such individuals, neonatal anti-inflammatory treatment may prove beneficial.

As discussed in Section 2, conventional second generation antipsychotics are not always successful at normalizing pro-inflammatory cytokines, illustrating the importance of evaluating the antiinflammatory effectiveness of antipsychotics. Various rodent studies have found haloperidol and other medications to be useful in reducing cytokine levels following bacterial/viral exposure. A maternal immune activation (MIA) model applied to rats found that treatment with haloperidol was effective in reducing IL-6 and TNF- $\alpha$ serum levels (Romero et al., 2007). By applying a similar MIA rat model, another study (Basta-Kaim et al., 2012) showed that chronic administration of either chlorpromazine or the second generation antipsychotic clozapine reduced the Prepulse Inhibition (PPI) deficit, a behavioural correlate for schizophrenia. Both antipsychotics also modulated enhanced levels of TNF- $\alpha$ as well as those of the cytokines IL-1 $\beta$ and IL-2.

Further research may lead to the discovery of new classes of antipsychotics which can be used in patients where the disease pathophysiology may be linked to prenatal or perinatal infection. For example, neonatal rodent models of schizophrenia have uncovered some potential novel antipsychotic treatments. Behavioural deficits were induced in rat pups through subchronic doses of epidermal growth factor (EGF) and Emodin, a broad tyrosine kinase inhibitor, was then found to eliminate PPI deficits after subchronic, oral administration (Mizuno et al., 2008). Similar results were achieved using a quinazoline derivative and ErbB1 inhibitor in another rodent model of the disease. This is a notable finding as abnormal ErbB1 signalling is thought to be a contributing factor to dopaminergic dysregulation in schizophrenia (Nawa and Mizuno, 2006). 
For patients believed to have been exposed to T.gondii, there is evidence that certain treatments may prove effective. The high levels of IgG antibodies against the parasite measured in both cerebrospinal fluid (CSF) and serum of drug naive schizophrenia patients, were found to be lower in recent-onset patients who had been receiving antipsychotic treatment (Leweke et al., 2004).

More detailed investigations into the types of antipsychotics which work well against T.gondii, conducted in vitro, found that two first-generation antipsychotics - fluphenazine and zuclopenthixol - have high anti-toxoplasma activity and three other first-generation medications - cyamemazine, levopromazine and loxapine - which are often prescribed to treat acute psychotic agitation, have intermediate anti-toxoplasma activity (Fond et al., 2014). Further research is needed to investigate the underlying molecular mechanisms behind their apparent effectiveness. This will also be required to verify whether these treatments could become either first-line antipsychotic medications for schizophrenia patients infected by T.gondii, or for use as add-on drugs to atypical antipsychotics.

\section{Antipsychotics and Anti-Inflammatory Medication for Schizophrenia}

\subsection{The potential benefits of add-on anti-inflammatory medication}

Anti-inflammatory agents may provide an effective means of lowering the proinflammatory status of some schizophrenia patients towards levels seen in healthy controls. Studies have shown that some antipsychotics have anti-inflammatory activity, but the immunomodulatory activities are generally very limited with the exception of clozapine (Leucht et al., 2013). Recent evidence suggests that the efficacy of antipsychotics could be augmented if patients were given additional anti-inflammatory medication in conjunction with standard antipsychotic treatment (Sommer et al., 2012). As heightened proinflammatory status can affect cognition by altering glutamatergic and dopaminergic pathways (Muller and Schwarz, 2006 and Muller and Dursun, 2011), restoring the balance between proinflammatory and anti-inflammatory regulators may improve cognitive symptoms.

To date, several anti-inflammatory medications have been examined as potential add-on treatments to antipsychotics. These include aspirin, celecoxib, cytostatics, davunetide, estrogens, fatty acids, leptin, tetracyclines, melatonin, minocycline, monoclonal antibodies, $\mathrm{N}$-acetyl cysteine (NAC), corticosteroids and drugs used in transplantation patients such as tacrolimus, cyclosporine and everolimus (Sommer et al., 2014).

\subsection{Evidence from clinical trials}

The results of clinical trials conducted so far have provided evidence that aspirin add-on to antipsychotic treatment reduces positive symptoms of schizophrenia (Laan et al., 2010), especially in patients in whom the disease duration has been relatively short. Aspirin is an non-steroidal antiinflammatory drug (NSAID) which inhibits the COX-1 enzyme and reduces inflammatory processes by altering the activity of $\mathrm{COX}-2$, thus reducing the production of thromboxanes and prostaglandins (Vane et al., 1998). A randomized, double-blind, placebo-controlled study of 70 patients in the Netherlands showed an improvement in positive and total PANSS scores after three months of daily $1000 \mathrm{mg}$ doses, but little change in cognitive function (Laan et al., 2010). However, the study did not examine the efficacy of aspirin over a longer time period.

Estrogens may also be a promising supplementary treatment for female patients (Kulkarni et al., 2008), although their effectiveness was greatly diminished when treating male schizophrenia 
patients (Kulkarni et al., 2011). They are thought to have mild anti-inflammatory activity mediated through various mechanisms of action including reducing levels of TNF- $\alpha$, Nitric Oxide and oxidative stress as well as influencing dopaminergic signalling (Liu et al., 2005).

The protein n-acetyl cycteine (NAC) has become of interest as a potential add-on treatment to antipsychotics as a result of the theory that antioxidant agents could be used to target the negative symptoms of schizophrenia. There is accumulating evidence for abnormalities in pathways involved with oxidative stress in the pathophysiology of schizophrenia (Wood et al., 2009). Compared to healthy controls, schizophrenia patients have been shown to have decreased levels of glutathione, superoxide dismutase (SOD), lower plasma nitrite concentrations and reduced red blood cell catalase levels in first-episode patients (Flatow et al., 2013). NAC is believed to be able to cross the BBB (Farr et al., 2003) and reach glial cells, initiating a cellular process which results in increased glutathione levels (Arakawa $M$ and Ito, 2007). Glutathione is an antioxidant which decreases cellular damage by scavenging reactive oxygen species (Müller and Dursun, 2011). NAC also has been shown to have direct free radical scavenging properties (Laan et al., 2009). In addition to its corrective effect on the oxidative balance, NAC possesses anti-inflammatory properties. It acts as a strong inhibitor on cytokines such as TNF- $\alpha$, IL- 6 and IL-1 $\beta$ which are the main mediators of proinflammatory status (Palacio et al., 2011). Furthermore, it is thought to exert beneficial regulatory effects on glutaminergic and dopaminergic pathways, neurotransmission pathways which are known to be altered in schizophrenia (Oja et al., 2000).

There have been a couple of studies (Berk et al., 2008 and Wood et al., 2009) which have attempted to assess the efficacy of NAC as an add-on medication to treat the negative symptoms of schizophrenia in chronic patients, by evaluating changes in PANSS scores. The initial study (Berk et al., 2008) found that $2 \mathrm{~g} /$ day of NAC in addition to various atypical antipsychotics over a 24 week period, resulted in significant improvements on the negative PANSS subscale as well as on the general and total subscales. The second study (Wood et al., 2009) took place over just eight weeks with a total of 42 patients. A smaller improvement in negative PANSS scores was reported. However this study only looked at patients who were in an active phase of their illness (patients with a score of 20 or more on the negative PANSS subscale were selected) which may explain the differences between the two studies.

Minocycline, davunetide, EPA and DHA fatty acids have also been evaluated in clinical trials but with relatively little success in augmenting the efficacy of antipsychotics. Celecoxib is a molecule which is able to cross the BBB (Davies et al., 2000) and blocks COX-2-mediated vascular permeability, preventing the extravasation of various pro-inflammatory molecules which mediate the inflammatory response (Simon, 1999). However the studies involving celecoxib have yielded mixed results, possibly due to the short duration of the trials (Muller et al., 2002, Rappard and Muller, 2004). Interestingly, celecoxib did show a strong positive effect when it was given solely to first episode psychosis (FEP) patients (Muller et al., 2005) so it may be of clinical use for recent-onset patients.

There is evidence that the level of the response to medication may also be strongly related to the initial inflammatory status. One study found that aspirin treatment yielded the greatest benefits in schizophrenia patients with a lower IFN- $/$ /IL-4 ratio (Laan et al., 2010) suggesting that the reduction of psychotic symptoms occurs to a greater extent among patients with relatively high levels of anti- 
inflammatory cytokines. Celecoxib was also found to work with a far greater efficacy in patients where the concentration of soluble TNF- $\alpha$ receptor 1 was lower (Muller et al., 2010).

\subsection{Limitations of studies conducted so far}

Due to the small number of studies conducted to date, it is difficult to draw any strong conclusions regarding the efficacy of the discussed anti-inflammatory agents for clinical use in schizophrenia. Aspirin, estrogens and NAC are the only compounds which have been shown to improve symptom severity but the mechanisms of action of these compounds are very different, thus it is unclear whether the positive clinical effect is derived from their anti-inflammatory actions or other mechanisms. While studies showed improvements in PANSS scores which were the primary means of assessment, they did not show corresponding improvements in cognition. Estrogens may improve symptoms by mediating angiotensin and dopamine transmission (Sanchez et al., 2012) and aspirin may do so by antagonizing dysfunction of the NMDA receptor (Goff and Coyle, 2001).

In addition, the side effect profile of using some of these drugs in conjunction with antipsychotics has yet to be carefully examined. It is believed that estrogens should not be taken for more than 1-2 months, unless taken with progesterone (Sommer et al., 2014). Long-term aspirin and celecoxib use can be associated with a substantial risk of gastrointestinal bleeding (Sommer et al., 2014). Celecoxib has also been associated with an increased cardiovascular risk (Solomon et al., 2006).

Early evidence suggests the low side effect profile of NAC may make it a preferable antiinflammatory agent compared to aspirin, estrogens and celecoxib. NAC's modulation of the cystineglutamate antiporter makes it effective at decreasing addiction behaviour, an additional benefit for patients. Improvements have been reported in cocaine addicts after treatment with NAC (Schmaal et al., 2012). However there have been very few studies so far investigating NAC's therapeutic efficacy in schizophrenia patients and those studies which have been conducted have investigated small patient cohorts over a short observational period. So far it is largely NSAIDs which have been investigated but there are a number of steroidal anti-inflammatory drugs which might be of interest, such as glucocorticosteroids which have been found to be effective in reducing the number of new psychotic episodes (Laan et al., 2009).

\section{Conclusion}

The studies discussed in this review are an attempt to highlight some of the most relevant research regarding the effects of antipsychotic medication on immune/inflammatory protein biomarkers which are frequently altered in patients.

Extensive studies have been conducted examining peripheral alterations in patients with the aim of detecting relevant subgroups and enhancing the quality of therapeutic interventions. Immune/inflammatory abnormalities represent some of the most robustly reported findings. While antipsychotic medication has proven efficacious in reducing the enhanced immune/inflammatory changes in some patients, this is not the case in all individuals. Trials investigating efficacy of antiinflammatory drug add-on to existing antipsychotics have obtained encouraging results. For example, aspirin and celecoxib yielded the greatest benefits in patients. Other promising results have been achieved for estrogens and NAC. However larger clinical trials need to be conducted over a longer duration in order to adequately assess their efficacy and side effect profiles. 
A different approach may be needed for patients who have been infected by microbial agents such as T.gondii. A series of first-generation antipsychotics have been found to have anti-toxoplasma properties but these studies have only been conducted in vitro. Clinical use may lead to problematic side effects such as EPS. Further research may be warranted into new compounds such as Emodin which has yielded promising results in preclinical rodent models, and specific anti-parasitic agents such as quinolones.

Finally, adopting a more individualized treatment approach by stratifying patients based on changes in biomarkers, will help to identify individuals who are more likely to respond to specific antipsychotic drugs and/or anti-inflammatory medications. Such an approach will also help to select the most effective treatments and avoid unnecessary side effects, to the benefit of both the patient and the healthcare service.

\section{References}

1. Tandon R, Keshavan M, Nasrallah $H$ (2008) "Schizophrenia, just the facts: What we know in 2008:Part 1: Overview." Schizophrenia Research. 100(1): 4-19.

2. Tomasik J, Rahmoune H, Guest PC, Bahn S (2014). Neuroimmune biomarkers in schizophrenia. Schizophr Res. S0920-9964(14)00382-X.

3. Potvin S, Stip E, Sepehry AA, Gendron A, Bah R, Kouassi E (2008). Inflammatory cytokine alterations in schizophrenia: a systematic quantitative review. Biol. Psychiatry, 63(8):801-808.

4. Chan MK, Guest PC, Levin Y, Umrania Y, Schwarz E, Bahn S, Rahmoune H (2011). Converging evidence of blood-based biomarkers for schizophrenia: an update. Int. Rev. Neurobiol. 101: 95-144.

5. Benros ME, Nielsen PR, Nordentoft M, Eaton WW, Dalton SO, Mortensen PB (2011). Autoimmune diseases and severe infections as risk factors for schizophrenia: a 30-year population-based register study. Am. J. Psychiatry. 168(12):1303-1310.

6. Smyth AM, Lawrie SM (2013). The neuroimmunology of schizophrenia. Clin Psychopharmacol Neurosci. 11(3):107-17.

7. Hwang Y, Kim J, Shin JY, Kim JI, Seo JS, Webster MJ, Lee D, Kim S (2013). Gene expression profiling by mRNA sequencing reveals increased expression of immune/inflammation-related genes in the hippocampus of individuals with schizophrenia. Transl Psychiatry. 3:e321

8. Schizophrenia Psychiatric Genome-Wide Association Study (GWAS) Consortium (2011). Genomewide association study identifies five new schizophrenia loci. Nat Genet. 43(10):969-76.

9. Drexhage RC, Weigelt K, van Beveren N, Cohen D, Versnel MA, Nolen WA, Drexhage HA (2011). Immune and neuroimmune alterations in mood disorders and schizophrenia. Int. Rev. Neurobiol., 101:169-201.

10. Watanabe $Y$, Someya T, Nawa $H$ (2010). Cytokine hypothesis of schizophrenia pathogenesis: evidence from human studies and animal models. Psychiatry Clin. Neurosci. 64(3):217-230. 
11. Simard AR, Rivest S (2005). Do pathogen exposure and innate immunity cause brain diseases? Neurol Res. 27(7):717-725.

12. Namba $H$, Nagano $T$, Iwakura $Y$, Xiong $H$, Jourdi $H$, Takei $N$, Nawa $H(2006)$. Transforming growth factor alpha attenuates the functional expression of AMPA receptors in cortical GABAergic neurons. Mol Cell Neurosci. 31(4):628-641.

13. Kirch DG, Alexander RC, Suddath RL, Papadopoulos NM, Kaufmann CA, Daniel DG, Wyatt RJ (1992). Blood-CSF barrier permeability and central nervous system immunoglobulin $G$ in schizophrenia. J Neural Transm Gen Sect. 89(3):219-232.

14. Miller BJ, Buckley P, Seabolt W, Mellor A, Kirkpatrick B (2011). Meta-analysis of cytokine alterations in schizophrenia: clinical status and antipsychotic effects. Biol. Psychiatry. 70(7):663-671. 15. Raison CL, Miller AH (2011). Is depression an inflammatory disorder? Curr Psychiatry Rep. 13(6):467-475.

16. Pae CU, Yoon CH, Kim TS, Kim JJ, Park SH, Lee CU, Lee SJ, Lee C, Paik IH (2006). Antipsychotic treatment may alter T-helper (TH) 2 arm cytokines. Int Immunopharmacol, 6(4):666-671.

17. Maes M, Bocchio Chiavetto L, Bignotti S, Battisa Tura G, Pioli R, Boin F, Kenis G, Bosmans E, de Jongh R, Lin A, Racagni G, Altamura CA (2000). Effects of atypical antipsychotics on the inflammatory response system in schizophrenic patients resistant to treatment with typical neuroleptics. Eur Neuropsychopharmacol. 10(2):119-124.

18. Buchholz S, Morrow AF, Coleman PL (2008). Atypical antipsychotic-induced diabetes mellitus: an update on epidemiology and postulated mechanisms. Intern Med J. 38(7):602-606.

19. Tourjman V, Kouassi É, Koué MÈ, Rocchetti M, Fortin-Fournier S, Fusar-Poli P, Potvin S (2013). Antipsychotics' effects on blood levels of cytokines in schizophrenia: a meta-analysis. Schizophr. Res. 151(1-3):43-47.

20. Lowe GD (2005). Circulating inflammatory markers and risks of cardiovascular and noncardiovascular disease. J. Thromb. Haemost. 3(8):1618-1627.

21. Dickerson F, Stallings C, Origoni A, Vaughan C, Khushalani S, Yang S, Yolken R (2013). C-reactive protein is elevated in schizophrenia. Schizophr Res. 143(1):198-202.

22. Miller BJ, Culpepper N, Rapaport MH (2014). C-reactive protein levels in schizophrenia: a review and meta-analysis. Clin. Schizophr. Relat. Psychoses. 7(4):223-230.

23. Diaz FJ, Pérez-Iglesias R, Mata I, Martínez-Garcia O, Vázquez-Barquero JL, de Leon J, CrespoFacorro B (2010). Possible effects of some antipsychotic drugs on C-reactive protein in a drug-naïve psychotic sample. Schizophr Res. 121(1-3):207-12.

24. De Witte L, Tomasik J, Schwarz E, Guest PC, Rahmoune H, Kahn RS, Bahn S (2014). Cytokine alterations in first-episode schizophrenia patients before and after antipsychotic treatment. Schizophr Res. 154(1-3):23-29. 
25. Dantzer R (2004). Cytokine-induced sickness behaviour: a neuroimmune response to activation of innate immunity. Eur. J. Pharmacol. 500(1-3):399-411.

26. Keller WR, Kum LM, Wehring HJ, Koola MM, Buchanan RW, Kelly DL (2013). A review of antiinflammatory agents for symptoms of schizophrenia. J. Psychopharmacol. 27(4):337-342.

27. Tappia PS, Troughton KL, Langley-Evans SC, Grimble RF (1995). Cigarette smoking influences cytokine production and antioxidant defences. Clin. Sci. (Lond.), 88(4):485-489.

28. Maes M, Song C, Lin A, De Jongh R, Van Gastel A, Kenis G, Bosmans E, De Meester I, Benoy I, Neels H, Demedts P, Janca A, Scharpé S, Smith RS (1998). The effects of psychological stress on humans: increased production of pro-inflammatory cytokines and a Th1-like response in stressinduced anxiety. Cytokine. 10(4):313-318

29. Murray RM, Lewis SW (1987). Is schizophrenia a neurodevelopmental disorder? Br Med J (Clin Res Ed). 295(6600):681-682.

30. Brown AS, Begg MD, Gravenstein S, Schaefer CA, Wyatt RJ, Bresnahan M, Babulas VP, Susser ES (2004). Serologic evidence of prenatal influenza in the etiology of schizophrenia. Arch Gen Psychiatry. 61(8):774-780.

31. Suvisaari J, Haukka J, Tanskanen A, Hovi T, Lönnqvist J (1999). Association between prenatal exposure to poliovirus infection and adult schizophrenia. Am J Psychiatry. 156(7):1100-1102

32. Brown AS, Cohen P, Greenwald S, Susser E (2000). Nonaffective psychosis after prenatal exposure to rubella. Am J Psychiatry. 157(3):438-443.

33. Babulas V, Factor-Litvak P, Goetz R, Schaefer CA, Brown AS (2006). Prenatal exposure to maternal genital and reproductive infections and adult schizophrenia. Am J Psychiatry. 163(5):927929.

34. Buka SL, Cannon TD, Torrey EF, Yolken RH; Collaborative Study Group on the Perinatal Origins of Severe Psychiatric Disorders (2008). Maternal exposure to herpes simplex virus and risk of psychosis among adult offspring. Biol Psychiatry. 63(8):809-815

35. Brown AS, Schaefer CA, Quesenberry CP Jr, Shen L, Susser ES (2006). No evidence of relation between maternal exposure to herpes simplex virus type 2 and risk of schizophrenia? Am J Psychiatry. 163(12):2178-2180.

36. Mortensen PB, Nørgaard-Pedersen B, Waltoft BL, Sørensen TL, Hougaard D, Torrey EF, Yolken RH (2007). Toxoplasma gondii as a risk factor for early-onset schizophrenia: Analysis of filter paper blood samples obtained at birth. Biol Psychiatry. 61(5):688-693.

37. Brown AS, Schaefer CA, Quesenberry CP Jr, Liu L, Babulas VP, Susser ES (2005). Maternal exposure to toxoplasmosis and risk of schizophrenia in adult offspring. Am J Psychiatry. 162(4):767773. 
38. Webster JP, Kaushik M, Bristow GC, McConkey GA (2013). Toxoplasma gondii infection, from predation to schizophrenia: can animal behaviour help us understand human behaviour? The Journal of experimental biology. 216(1):99-112.

39. Niebuhr DW, Millikan AM, Cowan DN, Yolken R, Li Y, Weber NS (2008). Selected infectious agents and risk of schizophrenia among U.S. military personnel. Am J Psychiatry. 165(1):99-106.

40. Amminger GP, McGorry PD, Berger GE, Wade D, Yung AR, Phillips LJ, Harrigan SM, Francey SM, Yolken RH (2007). Antibodies to infectious agents in individuals at ultra-high risk for psychosis. Biol Psychiatr. 61(10):1215-1217.

41. Leask SJ, Done DJ, Crow TJ (2002). Adult psychosis, common childhood infections and neurological soft signs in a national birth cohort. Br J Psychiatry. 181:387-392.

42. Parham P (2005). The immune system. Chaps. 1 and 3. 2nd ed. New York: Garland Science, Taylor \& Francis Group.

43. Richard MD, Brahm NC (2012). Schizophrenia and the Immune System. Am J Health Syst Pharm. 69(9):757-766.

44. Brown AS, Hooton J, Schaefer CA, Zhang H, Petkova E, Babulas V, Perrin M, Gorman JM, Susser ES (2004). Elevated maternal interleukin-8 levels and risk of schizophrenia in adult offspring. Am J Psychiatry. 161(5):889-895

45. Li Y, Ohls RK, Rosa C, Shah M, Richards DS, Christensen RD (1995). Maternal and umbilical serum concentrations of granulocyte colony-stimulating factor and its messenger RNA during clinical chorioamnionitis. Obstet Gynecol. 86(3):428-432

46. Banks WA, Kastin AJ, Gutierrez EG (1994). Penetration of interleukin-6 across the murine bloodbrain barrier. Neurosci Lett. 179(1-2):53-56

47. Dammann O, Leviton A (1997). Maternal intrauterine infection, cytokines and brain damage in the preterm newborn. Pediatr Res. 42(1):1-8.

48. Davis KL, Stewart DG, Friedman JI, Buchsbaum M, Harvey PD, Hof PR, Buxbaum J, Haroutunian V (2003). White matter changes in schizophrenia: evidence for myelin-related dysfunction. Arch Gen Psychiatry. 60(5):443-456.

49. Turnbull AV, Rivier CL (1995). Regulation of the HPA axis by cytokines. Brain Behav Immunity. 9(4):253-275.

50. Brown AS, Derkits EJ (2010) Prenatal Infection and Schizophrenia: A Review of Epidemiologic and Translational Studies. Am J Psychiatry. 167(3):261-280.

51. Girgis RR, Kumar SS, Brown AS (2014) The Cytokine Model of Schizophrenia: Emerging Therapeutic Strategies. Biological Psychiatry. 75(4):292-299.

52. Smith SE, Li J, Garbett K, Mirnics K, Patterson PH (2007) Maternal immune activation alters fetal brain development through interleukin-6. J Neurosci. 27(40):10695-10702. 
53. Romero E, Ali C, Molina-Holgado E, Castellano B, Guaza C, Borrell J (2007). Neurobehavioral and immunological consequences of prenatal immune activation in rats: Influence of antipsychotics. Neuropsychopharmacology. 32(8):1791-1804.

54. Gilmore JH, Jarskog LF, Vadlamudi S (2005). Maternal poly I:C exposure during pregnancy regulates TNF alpha, BDNF, and NGF expression in neonatal brain and the maternal-fetal unit of the rat. J Neuroimmunol. 159(1-2):106-12.

55. Amminger GP, McGorry PD, Berger GE, Wade D, Yung AR, Phillips LJ, Harrigan SM, Francey SM, Yolken RH (2007). Antibodies to infectious agents in individuals at ultra-high risk for psychosis. Biol. Psychiatry. 61(10):1215-1217.

56. Hayes LN, Severance EG, Leek JT, Gressitt KL, Rohleder C, Coughlin JM, Leweke FM, Yolken RH, Sawa A (2014). Inflammatory molecular signature associated with infectious agents in psychosis. Schizophr Bull. 40(5):963-72.

57. Yolken RH, Torrey EF, Lieberman JA, Yang S, Dickerson FB (2011). Serological evidence of exposure to Herpes Simplex Virus type 1 is associated with cognitive deficits in the CATIE schizophrenia sample. Schizophr. Res. 128(1-3):61-65.

58. Prasad KM, Watson AM, Dickerson FB, Yolken RH, Nimgaonkar VL (2012). Exposure to herpes simplex virus type 1 and cognitive impairments in individuals with schizophrenia. Schizophr. Bull. 38(6):1137-1148.

59. Dickerson F, Stallings C, Origoni A, Vaughan C, Khushalani S, Yolken R (2012). Additive effects of elevated C-reactive protein and exposure to Herpes Simplex Virus type 1 on cognitive impairment in individuals with schizophrenia. Schizophr. Res. 134(1):83-88.

60. Romero E, Ali C, Molina-Holgado E, Castellano B, Guaza C, Borrell J (2007). Neurobehavioral and Immunological Consequences of Prenatal Immune Activation in Rats. Influence of Antipsychotics. Neuropsychopharmacology. 32(8):1791-1804.

61. Basta-Kaim A, Szczęsny E, Leśkiewicz M, Głombik K, Slusarczyk J, Budziszewska B, Regulska M, Kubera M, Nowak W, Wędzony K, Lasoń W (2012). Maternal immune activation leads to age-related behavioral and immunological changes in male rat offspring - the effect of antipsychotic drugs.

Pharmacol Rep. 64(6):1400-10.

62. Mizuno $M$, Kawamura $H$, Takei N, Nawa $H$ (2008). The anthraquinone derivative Emodin ameliorates neurobehavioral deficits of a rodent model for schizophrenia. J. Neural. Transm. 115(3): 521-530.

63. Nawa H, Mizuno M (2006). Antipsychotic molecular-targeting epithelial growth factor receptor. US Patent US2006167026.

64. Leweke FM, Gerth CW, Koethe D, Klosterkötter J, Ruslanova I, Krivogorsky B, Torrey EF, Yolken $\mathrm{RH}$ (2004). Antibodies to infectious agents in individuals with recent onset schizophrenia. Eur. Arch. Psychiatry Clin. Neurosci. 254(1):4-8. 
65. Fond G, Macgregor A, Tamouza R, Hamdani N, Meary A, Leboyer M, Dubremetz JF (2014). Comparative analysis of anti-toxoplasmic activity of antipsychotic drugs and valproate. Eur Arch Psychiatry Clin Neurosci. 264(2):179-83.

66. Leucht S, Cipriani A, Spineli L, Mavridis D, Orey D, Richter F, Samara M, Barbui C, Engel RR, Geddes JR, Kissling W, Stapf MP, Lässig B, Salanti G, Davis JM (2013). Comparative efficacy and tolerability of 15 antipsychotic drugs in schizophrenia: a multiple-treatments meta-analysis. Lancet. 382(9896):951-962.

67. Sommer IE, de Witte L, Begemann M, Kahn RS (2012). Nonsteroidal anti-inflammatory drugs in schizophrenia: ready for practice or a good start? A meta-analysis. J Clin Psychiatry. 73(4):414-9.

68. Muller N, Schwarz M (2006). Schizophrenia as an inflammation-mediated dysbalance of glutamatergic neurotransmission. Neurotox Res. 10(2):131-148.

69. Müller N, Dursun SM (2011). Schizophrenia genes, epigenetics and psychoneuroimmunology therapeutics: all make sense now? J Psychopharmacol. 25(6):713-714.

70. Sommer IE, van Westrhenen R, Begemann MJ, de Witte LD, Leucht S, Kahn RS (2014). Efficacy of anti-inflammatory agents to improve symptoms in patients with schizophrenia: an update. Schizophr Bull. 40(1):181-91.

71. Laan W, Grobbee DE, Selten JP, Heijnen CJ, Kahn RS, Burger H (2010). Adjuvant aspirin therapy reduces symptoms of schizophrenia spectrum disorders: results from a randomized, double-blind, placebo-controlled trial. J Clin Psychiatry. 71(5):520-527.

72. Vane JR, Bakhle YS, Botting RM (1998). Cyclooxygenases 1 and 2. Annu Rev Pharmacol Toxicol. 38:97-120.

73. Kulkarni J, de Castella A, Fitzgerald PB, Gurvich CT, Bailey M, Bartholomeusz C, Burger H (2008). Estrogen in severe mental illness: a potential new treatment approach. Arch Gen Psychiatry. 65(8):955-960.

74. Kulkarni J, de Castella A, Headey B, Marston N, Sinclair K, Lee S, Gurvich C, Fitzgerald PB, Burger $H$ (2011). Estrogens and men with schizophrenia: is there a case for adjunctive therapy? Schizophr Res. 125(2-3):278-283.

75. Liu X, Fan XL, Zhao Y, Luo GR, Li XP, Li R, Le WD (2005). Estrogen provides neuroprotection against activated microglia-induced dopaminergic neuronal injury through both estrogen receptor- $\alpha$ and estrogen receptor- $\beta$ in microglia. J Neurosc Res. 81(5):653-665.

76. Wood SJ, Yücel M, Pantelis C, Berk M (2009). Neurobiology of schizophrenia spectrum disorders: the role of oxidative stress. Ann Acad Med Singapore. 38(5):396-401.

77. Flatow J, Buckley P, Miller BJ (2013). Meta-analysis of oxidative stress in schizophrenia. Biol. Psychiatry. 74(6):400-409. 
78. Farr SA, Poon HF, Dogrukol-Ak D, Drake J, Banks WA, Eyerman E, Butterfield DA, Morley JE (2003). The antioxidants alpha-lipoic acid and $\mathrm{N}$-acetylcysteine reverse memory impairment and brain oxidative stress in aged SAMP8 mice. J Neurochem. 84(5):1173-1183.

79. Arakawa M, Ito Y (2007). N-acetylcysteine and neurodegenerative diseases: basic and clinical pharmacology. Cerebellum. 6(4):308-314.

80. Laan W, Smeets H, de Wit NJ, Kahn RS, Grobbee DE, Burger H (2009). Glucocorticosteroids associated with a decreased risk of psychosis. J Clin Psychopharmacol. 29(3):288-90.

81. Palacio JR, Markert UR, Martínez P (2011). Anti-inflammatory properties of N-acetylcysteine on lipopolysaccharide-activated macrophages. Inflamm Res. 60(7):695-704.

82. Oja SS, Janáky R, Varga V, Saransaari P (2000). Modulation of glutamate receptor functions by glutathione. Neurochem Int. 37(2-3):299-306.

83. Berk M, Copolov D, Dean O, Lu K, Jeavons S, Schapkaitz I, Anderson-Hunt M, Judd F, Katz F, Katz P, Ording-Jespersen S, Little J, Conus P, Cuenod M, Do KQ, Bush Al (2008). N-acetyl cysteine as a glutathione precursor for schizophrenia-a double-blind, randomized, placebo-controlled trial. Biol Psychiatry. 64(5):361-368.

84. Davies NM, McLachlan AJ, Day RO, Williams KM (2000). Clinical pharmacokinetics and pharmacodynamics of celecoxib: a selective cyclo-oxygenase-2 inhibitor. Clin Pharmacokinet. 38(3):225-242.

85. Simon LS (1999). Role and regulation of cyclooxygenase-2 during inflammation. Am J Med. 106(5B):37S-42S.

86. Müller N, Riedel M, Scheppach C, Brandstätter B, Sokullu S, Krampe K, Ulmschneider M, Engel RR, Möller HJ, Schwarz MJ (2002). Beneficial antipsychotic effects of celecoxib add-on therapy compared to risperidone alone in schizophrenia. Am J Psychiatry. 159(6):1029-1034.

87. Rappard F, Muller N (2004). Celecoxib add-on does not have beneficial antipsychotic effects over risperidone alone in schizophrenia [abstract]. Neuropsychopharmacology. 29:S183-S241.

88. Müller N, Riedel M, Schwarz MJ, Engel RR (2005). Clinical effects of COX-2 inhibitors on cognition in schizophrenia. Eur Arch Psychiatry Clin Neurosci. 255(2):149-151.

89. Müller N, Krause D, Dehning S, Musil R, Schennach-Wolff R, Obermeier M, Möller HJ, Klauss V, Schwarz MJ, Riedel M (2010). Celecoxib treatment in an early stage of schizophrenia: results of a randomized, double-blind, placebo-controlled trial of celecoxib augmentation of amisulpride treatment. Schizophr. Res. 121(1-3):118-124.

90. Sánchez MG, Morissette M, Di Paolo T (2012). Effect of a chronic treatment with $17 \beta$-estradiol on striatal dopamine neurotransmission and the Akt/GSK3 signaling pathway in the brain of ovariectomized monkeys. Psychoneuroendocrinology. 37(2):280-291.

91. Goff DC, Coyle JT (2001). The emerging role of glutamate in the pathophysiology and treatment of schizophrenia. Am J Psychiatry. 158(9):1367-1377. 
92. Solomon SD, Pfeffer MA, McMurray JJ, Fowler R, Finn P, Levin B, Eagle C, Hawk E, Lechuga M, Zauber AG, Bertagnolli MM, Arber N, Wittes J; APC and PreSAP Trial Investigators (2006). Effect of celecoxib on cardiovascular events and blood pressure in two trials for the prevention of colorectal adenomas. Circulation. 114(10):1028-1035.

93. Schmaal L, Veltman DJ, Nederveen A, van den Brink W, Goudriaan AE (2012). N-acetylcysteine normalizes glutamate levels in cocaine-dependent patients: a randomized crossover magnetic resonance spectroscopy study. Neuropsychopharmacology. 37(9):2143-2152.

94. Dean O, Giorlando F, Berk M (2011). N-acetylcysteine in psychiatry: current therapeutic evidence and potential mechanisms of action. J Psychiatry Neurosci. 36(2):78-86. 